2002

\title{
Statistical Theory Of Asteroid Escape Rates
}

Charles Jaffé

Shane D. Ross

Martin W. Lo

Jerrold Marsden

David Farrelly

See next page for additional authors

Follow this and additional works at: https://researchrepository.wvu.edu/faculty_publications

\section{Digital Commons Citation}

Jaffé, Charles; Ross, Shane D.; Lo, Martin W.; Marsden, Jerrold; Farrelly, David; and Uzer, T., "Statistical Theory Of Asteroid Escape Rates" (2002). Faculty Scholarship. 686.

https://researchrepository.wvu.edu/faculty_publications/686 
Authors

Charles Jaffé, Shane D. Ross, Martin W. Lo, Jerrold Marsden, David Farrelly, and T. Uzer 


\title{
Statistical Theory of Asteroid Escape Rates
}

\author{
Charles Jaffé, ${ }^{1,2,3}$ Shane D. Ross, ${ }^{1,2}$ Martin W. Lo, ${ }^{1,2}$ Jerrold Marsden, ${ }^{1}$ David Farrelly, ${ }^{4}$ and T. Uzer ${ }^{1,5}$ \\ ${ }^{1}$ Control and Dynamical Systems Division 107-81, California Institute of Technology, Pasadena, California 91125 \\ ${ }^{2}$ Navigation and Flight Mechanics, Jet Propulsion Laboratory, Pasadena, California 91109-8099 \\ ${ }^{3}$ Department of Chemistry, West Virginia University, Morgantown, West Virginia 26506-6045 \\ ${ }^{4}$ Department of Chemistry, Utah State University, Logan, Utah 84322-0300 \\ ${ }^{5}$ Center for Nonlinear Sciences and School of Physics, Georgia Institute of Technology, Atlanta, Georgia 30332-0430
} (Received 1 February 2002; published 12 June 2002)

\begin{abstract}
Transition states in phase space are identified and shown to regulate the rate of escape of asteroids temporarily captured in circumplanetary orbits. The transition states, similar to those occurring in chemical reaction dynamics, are then used to develop a statistical semianalytical theory for the rate of escape of asteroids temporarily captured by Mars. Theory and numerical simulations are found to agree to better than $1 \%$. These calculations suggest that further development of transition state theory in celestial mechanics, as an alternative to large-scale numerical simulations, will be a fruitful approach to mass transport calculations.
\end{abstract}

DOI: 10.1103/PhysRevLett.89.011101

While large-scale chaos exists in the Solar System, it is sufficiently weak that the motions of most of the planets appear quite regular, at least on relatively short time scales [1]. In contrast, smaller bodies such as asteroids and comets, through their interactions with the planets and the Sun, can exhibit strongly chaotic motion. Nevertheless, the ability to predict the behavior of populations of these small but numerous objects is essential for understanding such problems as the evolution of both short- and long-range comets originating in the Kuiper Belt and the Oort Cloud, respectively [2], the dynamics of near-Earth asteroids [4,5], zodiacal and circumplanetary dust dynamics [3], and the gravitationally assisted transport of spacecraft [6]. The discovery of possible traces of a living organism in a Martian meteorite found in Antarctica [7] has stimulated investigations into the feasibility of viable microbes transporting through space [8] and illustrates the fundamental importance of understanding mass transport in the Solar System to theories of the origin of life. In this Letter we study the problem of computing average rates of asteroid escape from Mars because of its bearing on understanding the feasibility of transport of viable microorganisms between the two planets.

On its face it might seem that once individual orbits are known the problem is solved. However, deeper insights can be obtained by computing rates because these allow models of the evolution of populations of particles to be constructed. In principle, the computation of rates of mass transport can be accomplished by large numerical simulations in which the orbits of vast numbers of test particles are propagated in time including as many interactions as desirable [2]. However, such calculations are computationally demanding and it may be difficult to extract from them information about key dynamical mechanisms. They do have the considerable advantage, however, that a variety of nongravitational effects can easily be included, even if these destroy the Hamiltonian nature of
PACS numbers: 96.35. $-\mathrm{j}, 05.45 .-\mathrm{a}, 82.20 . \mathrm{Db}, 95.10 . \mathrm{Ce}$

the problem. In this Letter a complementary approach is developed that can be used provided that the problem is of autonomous Hamiltonian form. We first identify transition states that regulate mass transport through bottlenecks in phase space [9]. Knowledge of the transition state allows us to compute rates using a Rice-RamspergerKassel-Marcus(RRKM)-like approach similar to that developed in chemical dynamics [10]. Molecular RRKM theory is an essentially statistical approach in which it is assumed that all molecular configurations within the initial microcanonical ensemble are equally likely to react. In other words, the rate of intramolecular energy redistribution is rapid compared to the rate of reaction. As a consequence, the reaction rate can be expressed as the ratio of the flux across the transition state divided by the total volume of phase space associated with the reactants. We provide the first application of this statistical approach in celestial mechanics and obtain a very high level of agreement with numerical simulations in which individual orbits are integrated numerically. Our results suggest that the validity of RRKM theory might even be better in celestial mechanics than in chemical dynamics.

First of all we demonstrate the existence of transition states in phase space which serve as bottlenecks to transport in the restricted three body problem (RTBP) which describes a rather wide variety of interesting phenomena in the Solar System $[6,11,12]$. One such example, which demonstrates the equivalence to a chemical reaction, is provided in Fig. 1: The left panel in Fig. 1 illustrates the dynamics shown by Jupiter-family comets such as Oterma and Gehrels 3 which shuttle back and forth between rather complicated heliocentric orbits outside the orbit of Jupiter (exterior region) and orbits inside (interior region) [6]. During these transitions the comets are frequently captured temporarily by Jupiter. Because the interior orbits are typically close to a $3: 2$ resonance (three revolutions around the Sun during two periods of Jupiter) while those in the 


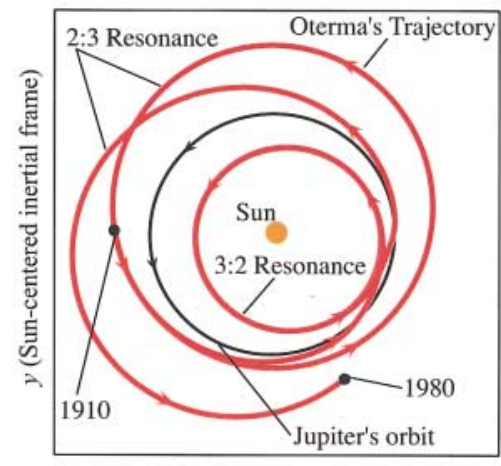

$x$ (Sun-centered inertial frame)

(a)

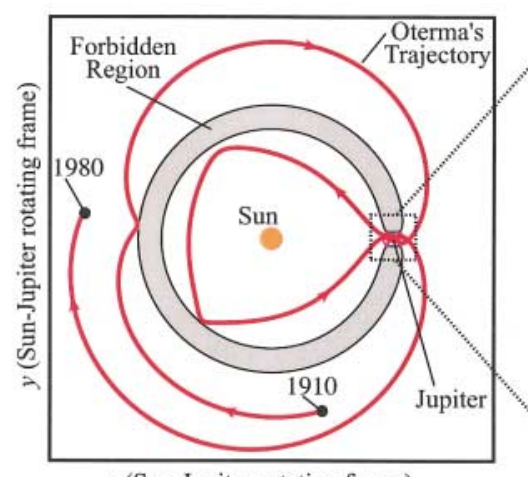

$x$ (Sun-Jupiter rotating frame)

(b)

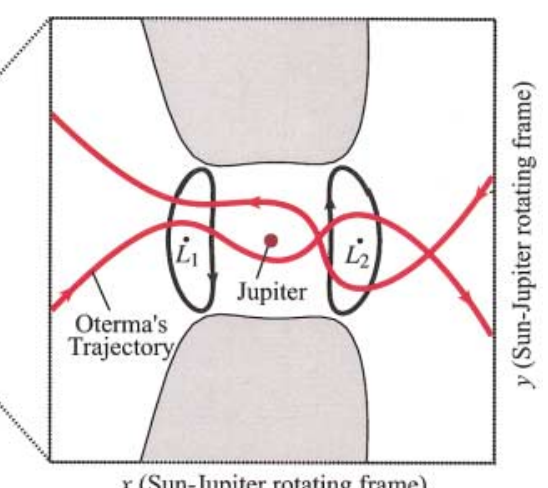

$x$ (Sun-Jupiter rotating frame)

(c)

FIG. 1 (color). Left panel: plot of a resonance switching orbit of comet Oterma in heliocentric coordinates. Middle panel: same as left panel in a coordinate system rotating with Jupiter. Right panel: expanded view of the bottleneck region.

exterior regions are near the 2:3 resonance the transitions shown in Fig. 1 are known as "resonance transitions." In noninertial coordinates, in a frame locked to the rotation of Jupiter, the picture is considerably clearer and the existence of the bottleneck region is readily apparent in the middle panel of Fig. 1. In fact, a dynamical invariant (the Jacobi integral - defined below) divides coordinate space into interior "reactant") and exterior ("product") regions which communicate through the narrow bottleneck containing Jupiter and two of the five Lagrange equilibrium points, $L_{1}$ and $L_{2}$. The passage of the comet through this region is regulated by phase space structures in the vicinity of the two saddle points $L_{1}$ and $L_{2}$-see Fig. 1. Indeed - and this is the key point of this Letter - the periodic orbits around the two saddles, which correspond to the transition states [13], control mass transport through the bottleneck. The identification of these structures allows us to compute average rates accurately without the need for large numerical simulations.

The RTBP Hamiltonian is

$$
\begin{aligned}
H= & E=\frac{1}{2}\left(p_{x}^{2}+p_{y}^{2}\right)-\left(x p_{y}-y p_{x}\right) \\
& -\frac{1-\mu}{r_{1}}-\frac{\mu}{r_{2}}-\frac{1}{2} \mu(1-\mu) .
\end{aligned}
$$

Here $E$ is the energy, $r_{1}=\sqrt{(x+\mu)^{2}+y^{2}}$, $r_{2}=\sqrt{(x-1+\mu)^{2}+y^{2}}$, and the masses for the Sun and the planet are $m_{S}=1-\mu$ and $m_{P}=\mu$. The coordinate system rotates about the center of mass with the Sun and the planet lying on the $x$ axis at $(-\mu, 0)$ and $(1-\mu, 0)$, respectively. The position of the third body (the asteroid) relative to the positions of the Sun and the planet is given as $(x, y)$. The Jacobi integral is

$$
\begin{aligned}
C(x, y, \dot{x}, \dot{y}) & =-\left(\dot{x}^{2}+\dot{y}^{2}\right)+2 \Omega(x, y) \\
& =-2 E(x, y, \dot{x}, \dot{y})
\end{aligned}
$$

$$
\begin{aligned}
\Omega(x, y)= & \frac{1}{2}\left(x^{2}+y^{2}\right)+\frac{1-\mu}{r_{1}} \\
& +\frac{\mu}{r_{2}}+\frac{1}{2} \mu(1-\mu) .
\end{aligned}
$$

Unlike most chemical reactions, this problem contains two saddle points with resonance transitions for Jupiter-family comets taking place when the Jacobi integral, $C$, is in the range $C_{3}<C<C_{2}$ with the subscripts referring to the values of $C$ at the similarly numbered Lagrange points. Then, the third body moves from the interior to the exterior of the orbit of Jupiter by passing through the narrow bottleneck in the vicinity of the planet. The presence of two equally important transition states makes this problem more intricate than is commonly found in chemical reaction dynamics where the flow is usually regulated by a single dominant transition state. By considering a different range of values of the Jacobi constant in the RTBP, however, we are able to find a direct analogy to the most well studied class of chemical reaction - unimolecular reactions. This occurs when $C_{2}<C<C_{1}$ and describes an asteroid (or any planetoid or particle) that is already orbiting a planet and which can escape inwards (towards the Sun) but not outwards, i.e., a particle trapped in the region near the planet can escape only through the bottleneck at $L_{1}$ shown in Fig. 1. We now specialize to asteroid escape from Mars orbits.

The general procedure for identifying the transition state(s) in two or more degrees of freedom using Hamiltonian normal form theory [14] is described in detail in Ref. [9]. Briefly, a sequence of local, nonlinear transformations of the coordinates is developed that transforms the Hamiltonian into the so-called normal form. In the Mars asteroid problem the linear part of the normal form in the neighborhood of the equilibrium point $L_{1}$ at $(k, 0)$ is

$$
H_{l}=\frac{1}{2}\left\{\left(p_{x}+y\right)^{2}+\left(p_{y}-x\right)^{2}-a x^{2}+b y^{2}\right\},
$$

where 
where $a=2 \rho+1$ and $b=\rho-1$ and where

$$
\rho=\mu|k-1+\mu|^{-3}+(1-\mu)|k+\mu|^{-3} .
$$

The $L_{1}$ equilibrium point is a saddle center [14]. Interestingly, in appropriate coordinates, this Hamiltonian is formally identical to that obtained in the problem of the ionization of a hydrogen atom in crossed electric and magnetic fields $[9,15,16]$. The transport of the electron from the interior region to the exterior region was shown to be regulated by a phase-space transition state $[13,17]$ associated with the periodic orbit centered on the equilibrium point at the Stark saddle - the atomic equivalent of $L_{1}$. This normal form expansion is valid in the neighborhood of the equilibrium point. Once the structure of phase space has been established locally, it can then be continued numerically outside of the local region. In the energy domain, this continuation is valid up to the next bifurcation of phase-space structure.

We consider a situation in which an asteroid (or other body) starts out in a circumplanetary orbit around Mars - perhaps the body arrived in orbit after it was ejected from the Martian surface following an impact. However they arrived in orbit, we are here only interested in the rate of escape of such bodies. Two sets of calculations were performed. The first set consists of direct Monte Carlo simulations of survival probabilities; 107000 particles with randomly selected initial conditions were started at 200 Mars radii from Mars and integrated until they escaped the planet by crossing the transition state and entered an orbit around the Sun. This ensemble is similar to a microcanonical ensemble in a chemical reaction in that the only restrictions on the initial conditions are due to energy constraints. The results of the simulations were binned and from this the rate was calculated directly. In the second set of computations (i) the transition state was identified, as described above and (ii) RRKM theory was used to determine the rate [10].

In RRKM theory the reaction rate, $k$, can be expressed as the ratio of the flux across the transition state divided by the volume of the portion of phase space associated with the reactants. Gray et al. have expressed this ratio as [18]

$$
\begin{aligned}
k= & \frac{1}{N_{A}} \int d R d P d \mathbf{r} d \mathbf{p} \delta(E-H) \\
& \times \delta\left(R-R^{\ddagger}\right) \theta(P)\left(\frac{P}{\mu}\right) .
\end{aligned}
$$

Here the integral is over all of phase space. The variables $R, P$ are the reaction coordinate and its conjugate momentum, while $\mathbf{r}, p$ refer to the bath coordinates (i.e., coordinates other than the reaction coordinate) and their conjugate momenta, and $R^{\ddagger}$ is the coordinate of the transition state. The first delta function restricts us to the energy shell and the second delta function restricts us to the phasespace transition state within that energy shell. This surface can be divided into two hemispheres characterized by the direction of the flow across the transition state [9]. The
Heaviside step function $\theta(P)$ selects the hemisphere with positive flux (from reactants to products). Thus, the integral in the numerator is the flux across the phase-space transition state. $N_{A}$ is essentially a normalization factor and in the simplest formulations is taken to be equal to the volume of the reactant region of the energy shell. The actual computations are done in Levi-Civita regularizing coordinates as described in Ref. [13] with the rate being thought of as the ratio of the flux that actually crosses the transition state to the flux attempting the transition, $\Phi_{\text {react }} / \Phi_{\text {total }}$.

The computations can be simplified by noting that, in two degree-of-freedom systems, the projection of an unstable periodic orbit into configuration space defines a surface that separates reactants and products. It is, therefore, called a "periodic orbit dividing surface" or PODS because, in phase space, the stable and unstable manifolds of this orbit partition the energy shell [9]. To compute $\Phi_{\text {total }}$ the PODS on the Mars side that has maximum flux is located. This PODS is then used to define the Poincare surface of section shown in Fig. 2. The chaotic flux, equal to the area of the chaotic sea, is evaluated by finding the "last" invariant tori bounding this sea. There are three of these, one for the outer shore, and two for the shores of the two inner islands. The sum and difference of the action integrals over these tori provide an excellent approximation to the area of the chaotic sea. The flux across the transition state, $\Phi_{\text {react }}$, is obtained by integration of the action integral over the PODS that has minimum flux over it.

Figure 3 shows the results of the two calculations for the initial rates, that is, after 40 orbital periods defined by the mean motion. After a brief initial period during

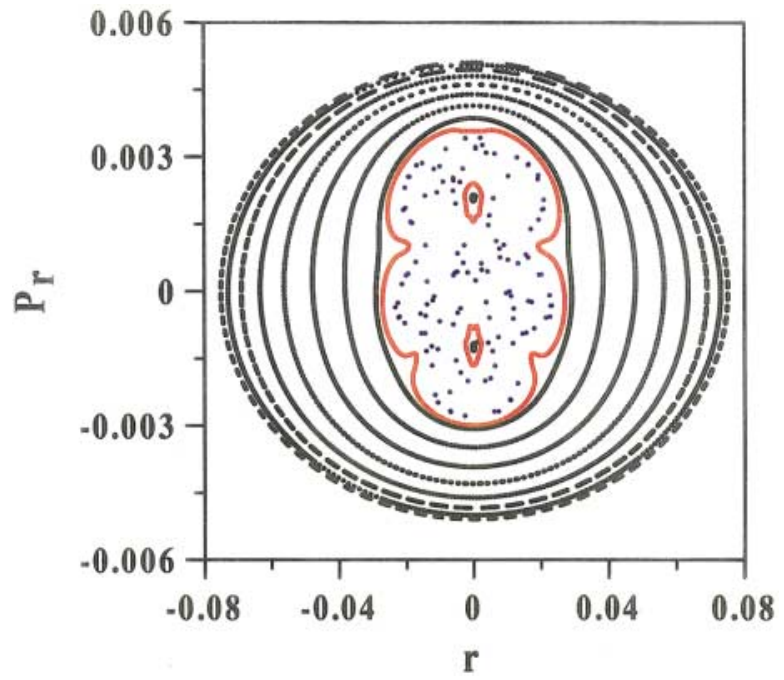

FIG. 2 (color). A composite surface of section (SOS) obtained by integration of 14 different trajectories. The SOS is defined using the PODS of maximum flux. The black points lie on invariant tori, while the blue points correspond to a single orbit that escapes after 228 periods. The red points correspond to the three trajectories which are confined to the last invariant tori defining the shores of the chaotic sea. 


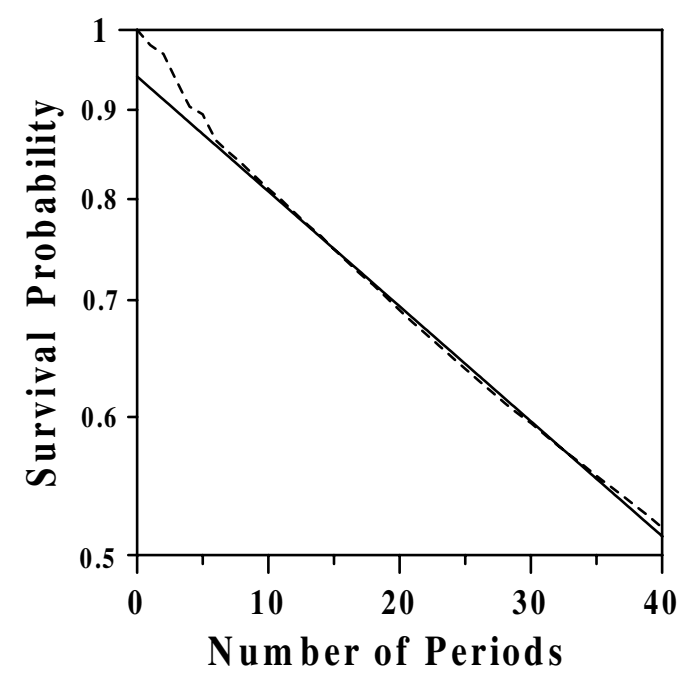

FIG. 3. Survival probability obtained from Monte Carlo (dashed line) and transition state theory (solid line) as described in the text. In the Monte Carlo calculations 107000 orbits were integrated using initial conditions chosen randomly from a one-dimensional manifold defined by a given value of the Jacobi integral $(C=-3.000936)$. After a short initial equilibration period the largest error settles down to on the order of $1 \%$ and error bars would not be visible on this plot. Scaled units are used.

which transients (due to details of the particular selection of initial conditions) die out, transition state theory settles down to agree to better than $1 \%$ with the numerical simulations. Non-RRKM behavior is the exception rather than the rule in chemistry, and our results suggest that in problems in the Solar System, the rate of the analogous mixing might also be fast compared to the rate of transition. Our calculations, which provide support for this assertion, will be tested further by extending our methods to higher dimensions [9]. In the asteroid escape problem studied here, the dynamics was confined to the plane so as to allow the simplest illustration of the method. However, the phase-space transition state theory is most powerful for multidimensional degree-of-freedom systems for which simulations become more difficult and insight into the dynamical mechanisms is harder to extract [9]. Examples include the evolution of long-range comets [2] and circumplanetary dust escape from nonequatorial "halo" orbits [19]. While we have not considered nongravitational forces our methods allow the inclusion, for example, of interactions of charged dust grains with planetary magnetic fields or the effect of solar radiation pressure.

This work was partly supported by the U.S. National Science Foundation, by the American Chemical Society (PRF), by the West Virginia-NASA Space Grant Program, and by NASA-ASEE (C. J.) This work was carried out in part at the Jet Propulsion Laboratory, California Institute of Technology, under a contract with the National Aeronautics and Space Administration.

[1] J. Laskar, Nature (London) 338, 237 (1989).

[2] P. Weigert and S. Tremaine, Icarus 137, 84 (1998).

[3] P. Michel, F. Migliorini, A. Morbidelli, and V. Zappalà, Icarus 145, 332 (2000).

[4] P. Farinella et al., Nature (London) 371, 314 (1994).

[5] M. Horányi, Annu. Rev. Astron. Astrophys. 34, 383 (1996).

[6] W. S. Koon, M. W. Lo, J. E. Marsden, and S. D. Ross, Chaos 10, 427 (2000).

[7] D. S. MacKay, E. K. Gibson, K. L. Thomas-Keprta, H. Vali, C.S. Romanek, S. J. Clemett, X. D. F. Chillier, C. R. Maechling, and R. N. Zare, Science 243, 924 (1996).

[8] C. Mileikowsky et al., Icarus 145, 391 (2000).

[9] S. Wiggins, L. Wiesenfeld, and C. Jaffé, and T. Uzer, Phys. Rev. Lett. 86, 5478 (2001).

[10] For reviews, see, e.g., J. C. Keck, Adv. Chem. Phys 13, 85 (1967); D. G. Truhlar, J. Phys. Chem. 100, 12271 (1996).

[11] R. Abraham and J. E. Marsden, Foundations of Mechanics (Addison-Wesley, New York, 1978), 2nd ed.

[12] K. E. Meyer and R. Hall, Hamiltonian Mechanics and the N-body Problem (Springer-Verlag, New York, 1992).

[13] C. Jaffé, D. Farrelly, and T. Uzer, Phys. Rev. Lett. 84, 610 (2000); Phys. Rev. A 60, 3833 (1999).

[14] V. I. Arnol'd, V. V. Kozlov, and A.I. Neishtadt, Mathematical Aspects of Classical and Celestial Mechanics (Springer-Verlag, New York, 1988).

[15] J. von Milczewski, D. Farrelly, and T. Uzer, Phys. Rev. Lett. 78, 2349 (1997).

[16] T. Uzer and D. Farrelly, Phys. Rev. A 52, R2501 (1995).

[17] S. Wiggins, Normally Hyperbolic Invariant Manifolds in Dynamical Systems (Springer-Verlag, New York, 1994).

[18] S. K. Gray, S. A. Rice, and M. J. Davis, J. Phys. Chem. 90, 3470 (1986).

[19] J. E. Howard, H. R. Dullin, and M. Horányi, Phys. Rev. Lett. 84, 3244 (2000). 